\title{
Bayesian Analysis of Zero-Truncated Poisson Model: Application to the Self-Controlled Case-series Design
}

\author{
Henry Athiany $^{1, *}$, Anthony Wanjoya ${ }^{1}$, George Orwa $^{1,2}$, Samuel Mwalili ${ }^{1}$ \\ ${ }^{1}$ Department of Statistics and Actuarial Sciences, Jomo Kenyatta University of Agriculture and Technology, Nairobi, Kenya \\ ${ }^{2}$ Deputy Principal, Bomet University College, Bomet, Kenya
}

\section{Email address:}

henry.athiany@jkuat.ac.ke (H. Athiany), awanjoya@fsc.jkuat.ac.ke (A. Wanjoya), gorwa@buc.ac.ke (G. Orwa), smusili@jkuat.ac.ke (S. Mwalili)

${ }^{*}$ Corresponding author

\section{To cite this article:}

Henry Athiany, Anthony Wanjoya, George Orwa, Samuel Mwalili. Bayesian Analysis of Zero-Truncated Poisson Model: Application to the Self-Controlled Case-series Design. International Journal of Data Science and Analysis. Vol. 6, No. 6, 2020, pp. 170-182.

doi: $10.11648 /$ j.ijdsa.20200606.12

Received: October 12, 2020; Accepted: October 28, 2020; Published: November 4, 2020

\begin{abstract}
A Bayesian Self-Controlled Case-Series (BSCCS) method is proposed and used to estimate the relative risk of an adverse drug event (ADE) given transient exposure to a drug or vaccine. Markov Chain Monte Carlo (MCMC) methods through WinBUGS are used to estimate parameters of the model given different settings and sample sizes. The method explores full posterior distribution for the model to obtain the relative risk estimates which at times is a challenge in likelihood analysis of complex models. Data was simulated for 10, 20 or 50 children aged between 365 and 730 days, and received their first dose of the measles, mumps, and rubella (MMR) vaccine within this follow-up period. Each child had the outcome event - viral-meningitis, in the follow-up period. Results of the data analysis indicated an increased risk of viral meningitis within 14-35 days post vaccination. Results of Bayesian approach are quite similar to the MLE risk estimates, assuming a non-informative prior. However, with more informative priors, BSCCS method produced better results with narrow credible intervals. For the real data, children aged 365 and 730 days, exposed to MMR vaccine, with viral meningitis (single exposure) were considered. While the frequentist approach estimated the incidence rate ratio (IRR) as IRR 12.037 (95\% CI (3.002 - 48.259)), the Bayesian estimate was IRR 8.971 (95\% CI 2.869 - 27.994). This is similar to the MLE results but with narrow credible intervals. In all cases, there is significantly higher risk of viral meningitis within 14-35 days post MMR vaccination. Results from the simulation study and real data revealed that the BSCCS model fitted better than the SCCS model.
\end{abstract}

Keywords: Zero-truncated Poisson Distribution, Case-series, Bayesian Self-controlled Case Series, MMR Vaccine, Viral Meningitis

\section{Introduction}

Truncated data arise when we restrict the range of possible outcomes of the data in some way [1]. The most common form of truncation that we often encounter is the omission of the zero class [2]. Zero-truncated count data models are often used, given the supported count distributions. These distributions, often assume the possibility of zero counts. Generally, zero-truncated data are not necessarily a problem, although quite common in health data. However, given the underlying assumptions of the supported count distributions; Hardin and Hilbe [3] pointed out that modeling such count data using regression methods that are based on non-truncated distributions is more likely to give biased results. This, becomes more likely when the mean response is closer to zero [3]. To deal with this problem of influence on inference from biased results, several methods of regression have been proposed by researchers to model such data for different situations [3]. These include among others, the Zero-Truncated Negative Binomial (ZTNB) and the Zero-Truncated Poisson (ZTP) regression. One of the modelling designs that employs the ZTP approach is the self-controlled case-series (SCCS) design [4].

SCCS is an innovative method developed by Whitaker, Paddy Farrington, Spiessens and Musonda [5] in the 
mid-1990s as an alternative to the bias-prone case-control or cohort studies. This method has been used to conduct robust studies in assessing the risk of adverse events given exposures to various health related time-varying exposures. Generally, the method is used to overcome the problem of between-person confounding. This is achieved by including in the study, only individuals with information on both exposure and outcome. This, therefore restricts individuals in the study to have had at least one outcome event [5].

\subsection{Zero-Truncated Poisson (ZTP) Distribution}

Consider $\mathrm{Y}$ to be a standard Poisson random variable with mean $\theta>0$ and the probability distribution being defined as

$$
f(y \mid \theta)=\frac{\theta^{y} e^{-\theta}}{y !}, y=0,1,2, \ldots
$$

The Poisson random $\mathrm{Y}$ in equation (1) has the mean, $\mathrm{E}(\mathrm{Y})=\theta$ and variance, $\operatorname{var}(\mathrm{Y})=\theta$. David and Johnson (1952) defined the density function of zero-truncated (or positive or conditional) Poisson distribution, that structurally excludes zero counts while modeling count data, as

$$
f(y \mid y>0, \theta)=\frac{\theta^{y} e^{-\theta}}{y !\left(1-e^{-\theta}\right)}, y=1,2,3, \ldots
$$

By conditioning equation (1) on $y>0$, that is, eliminating the possibility of the outcome zero from the sample space and re-normalizing the remaining density, David and Johnson [6] obtained equation (2). Later, while extending the work of David and Johnson [6], Tate and Goen [7] obtained the minimum variance unbiased estimator for the parameter of the left truncated Poisson distribution model, using the properties of sufficient statistics. Furthermore, the $\mathrm{r}^{\text {th }}$ factorial moment of $\mathrm{Y}$ in equation (2) as given by Johnson, Kemp and Kotz [2] is

$$
E[Y(Y-1) \ldots(Y-r+1)]=\left(1-e^{-\theta}\right)^{-1} \theta^{r}
$$

Hence, the basic parameters such as mean and variance of $\mathrm{Y}$ can be derived and given respectively as follows;

$$
E(Y \mid Y>)=\frac{\theta}{1-e^{-\theta}}=\frac{\theta e^{\theta}}{e^{\theta}-1}
$$

and variance

$$
\operatorname{var}(Y \mid Y>0)=\frac{\theta e^{\theta}}{e^{\theta}-1}\left\lceil 1-\frac{\theta}{e^{\theta}-1}\right\rceil=\frac{\theta\left(1-e^{-\theta}-\theta e^{-\theta}\right)}{\left(1-e^{-\theta}\right)^{2}}
$$

From the two results in equations (4) and (5), clearly, $\mathrm{E}(\mathrm{Y})<\mathrm{E}(\mathrm{Y} \mid \mathrm{Y}>0)$; while $\operatorname{var}(\mathrm{Y} \mid \mathrm{Y}>0)<\mathrm{E}(\mathrm{Y} \mid \mathrm{Y}>0)$, since $0<1-e^{-\theta}<1$, implying that $\operatorname{var}(\mathrm{Y} \mid \mathrm{Y}>0)$ is under-dispersed. To obtain the higher moments from this probability, we use the moment generating function ( $m g f)$ of the ZTP density. The mgf of the truncated Poisson mass function, $M_{T}(t)$ can be derived as follows;

$$
\begin{aligned}
M_{T}(t)=\mathrm{E}\left(e^{t y}\right)= & \sum_{y=1}^{\infty} e^{t y} \frac{e^{-\theta} \theta^{y}}{y !\left(1-e^{-\theta}\right)}=\frac{e^{-\theta}}{1-e^{-\theta}} \sum_{y=1}^{\infty} \frac{\left(\theta e^{t}\right)^{y}}{y !} \\
& =\frac{1}{1-e^{-\theta}}\left(e^{\theta\left(e^{t}-1\right)}-1\right)
\end{aligned}
$$

Using the $m g f$ in equation (7), the first central moment is obtained as follows

$$
\begin{gathered}
M_{T}^{\prime}(t)=\frac{M^{\prime}(t)}{1-e^{-\theta}} \\
E(Y)=\left.M_{T}^{\prime}(t)\right|_{t=0}=\frac{\theta}{1-e^{-\theta}}
\end{gathered}
$$

Likewise, the second and third central moments can be obtained for the mass function. Moreover, the cumulative distribution function can also be obtained as described by Springael and Van Nieuwenhuyse [8]. The model parameter estimation has previously been achieved either through maximum likelihood estimation (MLE) or by method of moments [9].

The ZTP model (2) can be extended to a regression model that accounts for the effect of independent variables. The mean parameter $\theta_{i}$ of the ZTP for the $i^{\text {th }}$ individual is usually linked to a linear predictor of $\mathrm{p}+1$ independent variables $X_{i}=\left(1, x_{i 1}, x_{i 2}, \ldots . x_{i p}\right)^{T}$ given the log link function, that is,

$$
E\left(y_{i} \mid X_{i}\right)=\theta_{i}=\exp \left(x_{i}^{\prime} \beta\right)
$$

or

$$
\log \left(\theta_{i}\right)=x_{i}^{\prime} \beta
$$

where $\beta=\left(\beta_{0}, \beta_{1}, \ldots, \beta_{p}\right)^{T}$ represents the unknown regression parameters. To ensure that $\theta_{i}$ is positive, for a proper distribution, the log link function is used. Thus, using Newton Raphson's method, parameter $\beta$ can be estimated by maximizing the log of ZTP likelihood [10]. The estimation procedure requires both the score and Hessian matrix which are obtained from the log-likelihood [11].

\subsection{Zero-Truncated Poisson Log-Likelihood}

Suppose $y_{1}, y_{2}, y_{3} \ldots y_{n}$ is an independent sample from equation (2), then the likelihood function is given by

$$
\mathcal{L}(\theta)=\mathcal{L}(\theta \mid y)=\prod_{i=1}^{n} \frac{e^{-\theta} \theta_{i}}{y_{i} !\left(1-e^{-\theta}\right)}=\frac{\theta^{\Sigma y_{i}} e^{-n \theta}}{\left(\Pi y_{i} !\right)\left(1-e^{-\theta}\right)^{n}}
$$

And the log-likelihood function is

$$
\begin{gathered}
\log \mathcal{L}(\theta)=\sum_{i=1}^{n}\left\{y_{i} \log (\theta)-\theta-\log \left(1-e^{-\theta}\right)-\log \left(y_{i}\right)\right\} \\
=\sum_{i=1}^{n}\left\{y_{i} \log (\theta)-\theta-\log \left(1-e^{-\theta}\right)-\log \Gamma\left(y_{i}+1\right)\right\}
\end{gathered}
$$

Since $\theta_{i}=\exp \left(x_{i}^{\prime} \beta\right)$ as given in equation (9), the log-likelihood function in equation (11) can therefore be expressed as;

$$
\log \mathcal{L}(\beta)=\sum_{i=1}^{n}\left\{y_{i} x_{i}^{\prime} \beta-\exp \left(x_{i}^{\prime} \beta\right)-\log \left(1-e^{-\exp \left(x_{i}^{\prime} \beta\right)}\right)-\log \Gamma\left(y_{i}+1\right)\right.
$$

To estimate the parameters, we maximize the log-likelihood

function. Moreover, the score function of equation (12) is 
obtained by taking the first derivative of the equation with respect to $\beta$. Similarly, taking the second partial derivatives gives us the Hessian matrix of the regression parameters as illustrated by Lee [11].

\section{The Self -Controlled Case Series (SCCS) Model}

Over the years, there has been an increasing availability of large scale longitudinal observational databases, which has provided opportunities for different approaches in data analysis. Despite this, the complexity and scale of some of the available databases presents some interesting statistical and computing challenges. The SCCS model was developed from a cohort study by conditioning on the occurrence of at least one event during the specified observation period of the individual, given an individual's history. The method models the event rate during exposure periods, in comparison to the baseline event rate while unexposed [4, 5].

In SCCS model, each individual act as their own control [4, 5]. An observation period is defined beforehand, and each treatment observation, which is a period of time that someone is exposed, is considered with respect to other periods of time in which the same person is not exposed. This way of matching, gracefully avoids patient-level selection bias $[4,5]$. Additionally, it helps to control for all fixed confounders, such as the individual's underlying frailty, the severity of their underlying disease, genetics, and socioeconomic status, amongst others. Figure 1 illustrates a typical example of the SCCS design. The figure shows the start of observation period, the risk period, event date - that can happen at any time within the follow-up period -, and the end of observation period. Furthermore, time varying covariate, age, has been partitioned into some age groups within the observation period. The choice of age group cut points depends on factors such as duration of study period, event of interest amongst others.

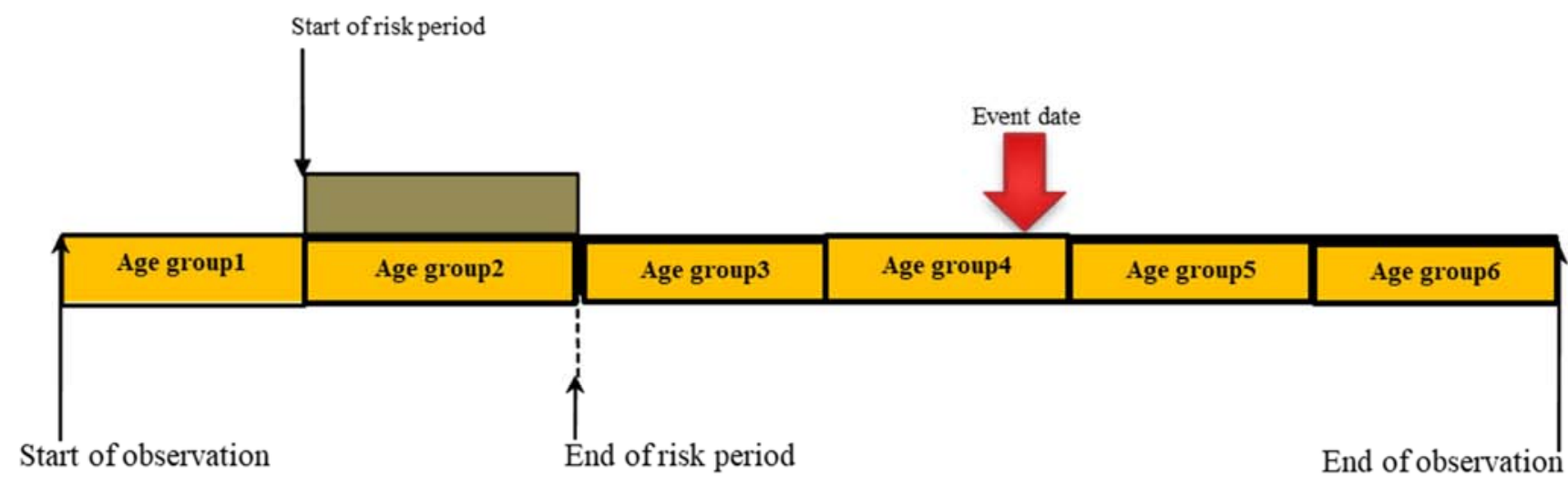

Figure 1. Graphical representation of self-controlled case series design.

Assuming that the outcome of interest arise in a non-homogeneous Poisson process, then the baseline incidence of the event for subject $i$ can be denoted by $e^{\psi_{i}}$, in age group $j$; while $e^{\alpha_{1}}$ and $e^{\beta_{1}}$ are the risk incidence associated with age groups $j=1$ and exposure period $k=1$ respectively relative to the baseline periods, $j=0$ and $k=0$ $[4,5]$. Building on this information, the general model based on a multinomial formulation will be as follows.

Consider a random selection of $n$ events in $N$ subjects $i=1,2,3 \ldots . N$ each with one or more events. Suppose $n_{i}$ denotes the number of events observed for subject $i$, implying $n=\sum_{i} n_{i}$. The observation period for subject $i$ can be split into intervals for age groups indexed by $j$ and risk periods indexed by $k$.

Taking $t_{i j k}$ to denote the time spent by subject $i$ in the age group $j$ and risk period $\mathrm{k}$, the incidence $\theta_{i j k}$ is assumed to be constant within each interval. A multiplicative model for the incidence function of the form in equation (13), can be assumed.

$$
\theta_{i j k}=e^{\zeta_{i}+\phi_{j}+\psi_{k}}
$$

or

$$
\log \left(\theta_{i j k}\right)=\zeta_{i}+\phi_{j}+\psi_{k}
$$

where $\zeta_{i}$ represents an effect for each subject $i, \phi_{j}$ represents an age group effect $j$, and $\psi_{k}$ represents an effect for risk group $k$.

The number of events $n_{i j k}$ is taken as the response variable. Thus, the associated main effects model will be:

$$
\begin{gathered}
n_{i j k}=\operatorname{Poisson}\left(\theta_{i j k} t_{i j k}\right) \\
\log \left(\theta_{i j k}\right)=\zeta_{i}+\phi_{j}+\psi_{k}
\end{gathered}
$$

where the $\log$ of the time spent in the interval $\ln \left(y_{i j k}\right)$ is included as an offset.

\section{Bayesian Inference for Zero-Truncated Poisson Count Data}

Bayesian approach considers prior information on the distribution of parameters, together with the likelihood of the observed data to construct posterior distribution of relevant quantities, for inference about the unknown parameters as well as other features of interest like data, or a combination 
of parameters [12]. The challenge in Bayesian statistics is choosing appropriate priors for a parameter. For convenience, conjugate priors are chosen to ensure that the resulting posterior distribution is in the same distributional family as the prior [13]. This may not be the case in many problems involving Bayesian analysis, especially when there are several parameters involved, for instance, a ZTP model. As a result, deriving the joint posterior distribution using standard densities may be challenging. However, simulation methods offer feasible strategies in such cases. For instance, in the absence of prior information on the parameter $\theta$ (equation. 2), using the uniform prior for $\theta$ will be appropriate as it is dominated by the likelihood. This, however, results in estimates that are similar to the results of frequentist approach [12].

\subsection{Bayesian ZTP Model Without Covariates}

For the parameter $\theta$ in equation (2), as a random variable, the gamma distribution is a natural conjugate of its prior distribution, which is given as

$$
p(\theta \mid \alpha, \beta)=\frac{\beta^{\alpha}}{\Gamma \alpha} \theta^{\alpha-1} e^{-\beta \alpha}, \alpha, \beta, \theta>0
$$

That is;

$$
p(\theta \mid \alpha, \beta) \propto \theta^{\theta-1} e^{-\beta \theta}
$$

Applying the Bayes theorem, the posterior distribution of $\theta$ from the likelihood function in (10) and the prior

$$
\mathcal{L}(\beta \mid y)=\prod_{i=1}^{n} \frac{\exp \left(x^{\prime} \beta\right)^{y_{i}} e^{\exp \left(x^{\prime} \beta\right)}}{y !\left(1-e^{\exp \left(x^{\prime} \beta\right)}\right)^{n}}=\frac{\exp \left(x^{\prime} \beta\right)^{\Sigma y_{i}} e^{-n\left(\exp \left(x^{\prime} \beta\right)\right.}}{\left(\prod y_{i} !\right)\left(1-e^{\exp \left(x^{\prime} \beta\right)}\right)^{n}}
$$

The prior is now given as $p(\beta) \sim M V \mathrm{~N}(\beta, \Sigma)$, that is;

$$
p(\beta)=(2 \pi)^{-\frac{1}{2}}(\operatorname{det} \Sigma)^{-\frac{1}{2}} \exp \left\{-\frac{1}{2}\left\{\beta-\beta_{0}\right\}^{\prime} \Sigma^{-1}\left\{\beta-\beta_{0}\right\}\right\}
$$

hence,

$$
p(\beta) \propto \exp \left\{-\frac{1}{2}\left\{\beta-\beta_{0}\right\}^{\prime} \Sigma^{-1}\left\{\beta-\beta_{0}\right\}\right\}
$$

Once again applying the Bayes theorem, the posterior distribution of $\theta$ from the likelihood function in (19) and the prior distribution function in (20) can now be shown to be

$$
p(\theta \mid y) \propto \mathcal{L}(\theta) p(\beta) \propto \frac{\exp \left(x^{\prime} \beta\right)^{\Sigma y_{i}} e^{-n\left(\exp \left(x^{\prime} \beta\right)\right)}}{\left(1-e^{-\exp \left(x^{\prime} \beta\right)}\right)^{n}} * \exp \left\{-\frac{1}{2}\left\{\beta-\beta_{0}\right\}^{\prime} \Sigma^{-1}\left\{\beta-\beta_{0}\right\}\right\}
$$

\section{Sampling Technique}

Given the challenges in evaluating the posterior distribution in equations (18) and (22) analytically, simulation-based Markov Chain Monte Carlo (MCMC) techniques are used to draw independent samples from the target distribution. The ability to sample from the posterior distribution is therefore essential as it permits Monte Carlo estimation of all posterior quantities of interest. To achieve this, Gibbs sampler, also known as alternating conditional sampling is used [12, 14].

Gibbs sampling is the second mechanism - after
Metropolis-Hastings (M-H) algorithm - that allows one to sample from the posterior distribution whenever direct approach is not possible. Basically, the idea is to split the multidimensional parameter vector $\theta$ into $d$ different blocks, $\theta=\theta_{j} \ldots \theta_{d}$ then sample from each block separately, conditioning on the most recent values of the remaining blocks [12]. Sampling of each block is randomly changed at each iteration. Full conditionals are used for construction of the Markov chain moves. This, eventually ensures that no rejection is experienced at any of the sampling steps.

Given the vector containing all parameters, $\theta=$ $\left(\theta_{j} \ldots . \theta_{d}\right)^{\prime}$ the Markovian updating scheme proceeds as 
follows;

Set $t=0$, and choose an arbitrary initial value of $\theta^{0}=$ $\left(\theta_{j}^{0}, \ldots \ldots \ldots . . . \theta_{d}^{0}\right)$

Generate each component of $\theta$ as follows

Draw $\theta_{1}^{(j+1)}$ from the full conditional

$$
p\left(\theta_{1} \mid \theta_{2}^{j}, \theta_{3}^{j} \ldots . \theta_{d}^{j}\right)
$$

Draw $\theta_{2}^{(j+1)}$ from the full conditional

$$
p\left(\theta_{2} \mid \theta_{1}^{j}, \theta_{3}^{j} \ldots . . \theta_{d}^{j}\right)
$$

Draw $\theta_{\mathrm{d}}^{(j+1)}$ from the full conditional

$$
p\left(\theta_{d} \mid \theta_{1}^{j}, \theta_{2}^{j} \ldots . . \theta_{d-1}^{j}\right)
$$

This completes one iteration of the Gibbs sampler, thereby producing one draw $\theta^{(j+1)}$.

Set $j=j+1$. If $\mathrm{j}<\mathrm{N}$, the desired sample size, then return to step (2), else stop.

\section{Sample Data Set, Exposure and Outcome of Interest}

The data set used in this study consists of children aged between 12 and 24 months who had a single exposure to Measles, Mumps and Rubella (MMR) Vaccine. More details on the data set is provided in section 6.2. The outcome of interest is viral meningitis. The following is a brief summary of the exposure and outcome of interest in the study.

\subsection{Measles, Mumps and Rubella Diseases}

Measles is a contagious disease caused by a single-stranded, negative-sense nonsegmented Ribonucleic Acid (RNA) virus of the genus Morbillovirus [15]. Measles is an airborne disease that easily spreads through contact with saliva or nasal secretions, cough or sneezes of an infected individual [15]. Ninety percent $(90 \%)$ of individuals who are not yet immune to the disease and are living with infected persons eventually catch the disease. The incubation period of the disease is 10-14 days (range 7-23 days). However, studies have shown that the virus easily becomes inactive under extreme $\mathrm{pH}$, heat or sunlight [16]. Serious complications of the disease includes amongst others, pneumonia and death. Although recognized as a disease of many years, studies have shown that with the introduction of the measles vaccine [17], the burden of the disease has been substantially reduced [18]. Moreover, almost all children vaccinated between 12 and 15 months usually develop the requisite antibodies [16].

Like measles, mumps is an acute viral disease caused by an enveloped, negative sense RNA virus of the genus Rubulavirus [19]. The disease is transmitted via direct contact with respiratory secretions, droplets nuclei or fomites that are transferred from the nose to mouth. Children under 5 years more commonly manifest symptoms of lower respiratory disease, while adults mostly experience asymptomatic infections [16]. World over, since the introduction of the mumps vaccine, the incidence of mumps infections witnessed a reduction to nearly $1 \%$ of the population per year by 1994 [17]. The reduction continues to be witnessed to date.

Rubella (German measles) on the other hand, is caused by an enveloped, positive-sense RNA togavirus of the genus Rubivirus [20]. The disease mainly spreads through contact with infectious respiratory secretions. If one is affected by the disease during pregnancy, then this may lead to congenital rubella infection in neonates [21]. In the early 1700 s, the disease was considered a disease of children and young adults. Currently, more than 100,000 cases world wide of infant rubella syndrome are witnessed yearly. Once infected by Rubella, one eventually becomes immune to the disease.

\subsection{Measles, Mumps, and Rubella - Vaccine and Some of the Side-effects}

The MMR vaccine is a safe and an effective vaccine used in preventing MMR diseases [17]. The vaccine is usually a mixture of live attenuated viruses of the three diseases. The first dose of the MMR vaccine injection is usually given to children at the ages 9 to 15 months. The second dose usually comes between 15 months to 6 years but with a gap of at least 4 weeks between two doses. Studies have indicated that 2-5\% of the children immunized with the first dose, fail to develop immunity to measles [22]. Hence, the second dose helps to produce this immunity that may have been missed in the first dose. A third dose may be recommended for groups of persons identified by public health authorities, especially during an outbreak [23, 24]. Like any other vaccine, some of the side effects that have been witnessed with MMR vaccine include; sore throat, fever and mild-rash. Previous studies have associated viral meningitis with exposure to MMR vaccines containing the Urabe strain of mumps [7, 25-27]. Moreover, research has also shown that children below 7 years old, have increased risk of febrile seizures 6 to 14 days after MMR vaccination. The vaccine has also been associated with increased incidence of ITP [28], although this is said to resolve after a period of seven days [28] or six months for acute conditions.

\subsection{Viral Meningitis}

Prior to the introduction of MMR vaccine, mumps virus was known as the main cause of viral (or aseptic) meningitis. Meningitis is an inflammation of the lining of the brain and the spinal cord [29]. It is mainly caused by a viral, bacterial or fungal infection. Viral meningitis is a viral disease that can attack people of all ages [30]. However, children below 5 years and individuals with weak immune systems are usually at a higher risk of contracting the disease. Despite being the most common form of meningitis, viral meningitis is less severe as compared to bacterial meningitis. The disease is 
more likely to develop to severe illness when one is less than 1 year old or has a very weak immune system [29]. Apart from mumps virus, some of the viruses that can cause viral meningitis include; Non-polio enteroviruses, Measles virus, Influenza virus, West Nile virus and Lymphocytic choriomeningitis virus [29]. Individuals with mild cases of viral meningitis usually recover fully within 7 to 10 days without any treatment. To prevent viral meningitis, one can be vaccinated against some of the diseases that lead to meningitis such as measles, mumps, chickenpox, and influenza. MMR vaccine is usually administered to children for this purpose.

The work presented here, extends the analysis that has been carried out in the previous studies [5], by considering in addition, a zero-truncated Bayesian case-series approach to investigate the association between MMR vaccine with viral meningitis hospital admission [29]. Empirical analysis is based on hospital data from Oxford, initially analyzed by Whitaker, Paddy Farrington, Spiessens and Musonda [5] using the frequentist SCCS framework.

\section{Simulation Study: Design and Analysis}

For the simulation process, data was generated where the true population value of the relative risk was assumed to be known. Bayesian Self-Controlled Case-Series (BSCCS) model was then used to analyze the simulated data and compare the value with the initially assumed population parameter value. This, helped in evaluating the performance of the proposed model. The simulation set-up mirrored what commonly happens with studies involving adverse drug events, such as vaccines. The same model was later fitted to the real data sets of MMR vaccine, with the ADE being viral meningitis [5].

\subsection{Simulation Setting}

The Oxford MMR vaccine data set described by Whitaker, Paddy Farrington, Spiessens and Musonda [5] on MMR vaccine, was used to set-up the simulation study. This, followed the following procedure. First, an observation period was declared as between 366 and 730 days after the birth of the child. As per MMR vaccination guidelines [17], this is the period over which a child is expected to receive the first dose of MMR vaccine. Three population relative risk values were assumed as 2.5, 5.0 and 12.04. The relative risk value 12.04 is similar to the results that have previously been presented for the MMR data set using a frequentist approach [5]. Three samples sizes of 10, 20 or 50 individuals were used to generate data for each of the population risk values. Time varying factors generated were outcome (event) date and the exposure date. Every child included in the study had an outcome event and must have also been exposed. An event date was assumed to occur at any time within the observation period. Since the start and end of observation period was fixed for this study, only the exposure (vaccination) and event dates were generated as follows. To generate the exposure date, a uniform distribution was assumed, with parameters, $\operatorname{runif}(n, 366,730)$, with $n$ being the sample size. Similarly, the event date was also assumed to follow a uniform distribution within the observation period, but taking into account, the assumed population relative risk. All $\mathrm{n}$ children had an exposure and just one outcome event as per the study design (Figure 1). This simulation approach is similar to the approach used by [31].

\subsubsection{Overview of the Simulation Setting}

Figure 2 gives a summary of the data simulation and analysis given the different sample sizes and for different population values assumed. First, set a seed, sample size and established the parameters required for the model. This was followed by generation of the exposure and outcome points. Finally, data management and analysis was carried out.

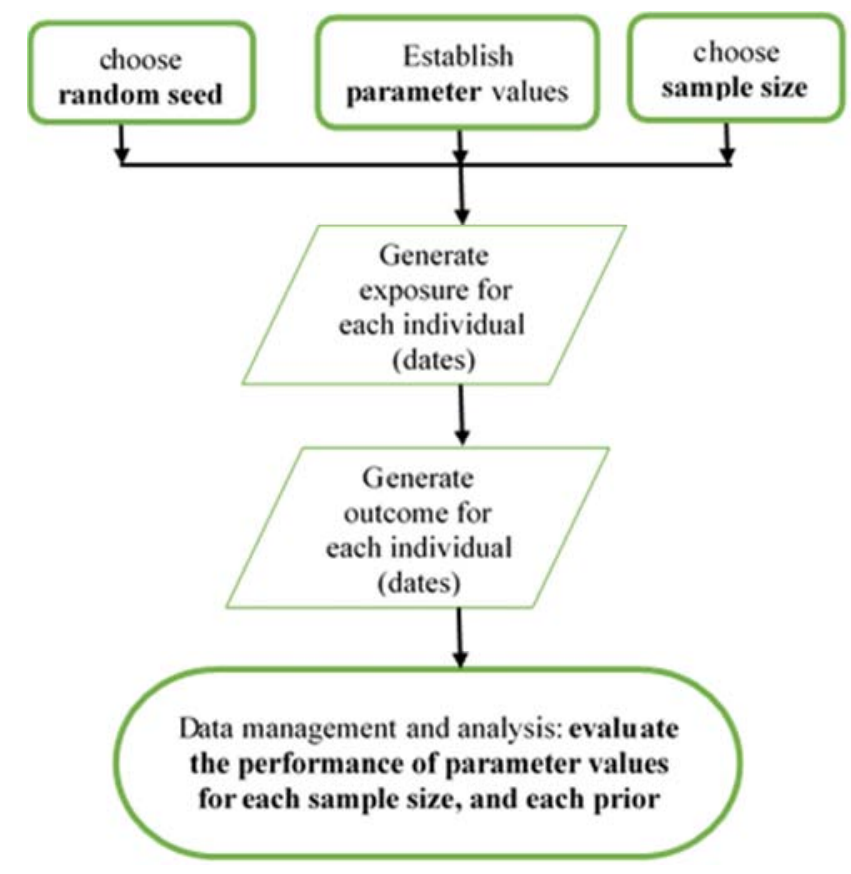

Figure 2. Summary of data generation and analysis for the simulation study.

\subsubsection{Sample of Simulated Dataset}

To simulate a sample data set, a population relative risk of 12.04 was assumed, sample size of 10 children, an observation period beginning 365 to 730 days after birth of the child. The MMR vaccine was assumed to be administered any time within the observation period and an event (viral meningitis) occurring anytime during the observation period, Table 1 displays a sample data set simulated, and used in this paper. There are two columns pre-risk and risk-end, that were included in the table after the data management process as detailed in section 6.1.3. To assess the performance of the proposed model, other data sets were simulated with the following combinations; sample sizes 10, 20 and 50, assumed population risk of $12.04,5$ and 2.5. 
Table 1. Simulated MMR vaccine data.

\begin{tabular}{|c|c|c|c|c|c|c|}
\hline child & pre-start & ex.day & pre-risk & risk-end & event.day & end.day \\
\hline 1 & 365 & 460 & 474 & 481 & 500 & 730 \\
\hline 2 & 365 & 500 & 514 & 521 & 520 & 730 \\
\hline 3 & 365 & 707 & 721 & 730 & 637 & 730 \\
\hline 4 & 365 & 435 & 449 & 470 & 450 & 730 \\
\hline 5 & 365 & 398 & 412 & 433 & 407 & 730 \\
\hline 6 & 365 & 704 & 728 & 730 & 720 & 730 \\
\hline 7 & 365 & 416 & 430 & 451 & 430 & 730 \\
\hline 9 & 365 & 592 & 592 & 613 & 565 & 730 \\
\hline 10 & 365 & 432 & 432 & 453 & 431 & 730 \\
\hline
\end{tabular}

\subsubsection{Management of Simulated Data Sets}

Ten individuals with ten outcomes were simulated following the procedure illustrated in section 6.1 as shown in Table 1. Variables included in the table are;

1. child-identifier for each child selected in the study

2. pre-start - age (days) just a day before the start of observation period

3. ex.day - age (days) when the child was exposed (received vaccination)

4. pre-risk - age (days) just before the beginning of the risk period

5. risk-end - age (days) last day of the risk period

6. event.day - age (days) that the outcome event was recorded

7. end.day - age (days) indicating the last day of the observation period

Since these children were followed for the same duration and same calendar period, the pre-start and end.day was the same for all ten of them, although this is not always a requirement. As highlighted (bold), child 2, 4, 7 and 8 had the event.day recorded during the risk period, while child 3, 6, 9 and 10 had the event.day coming before they were vaccinated. Given that the risk of viral meningitis varies with age, like the case of [5], only two age groups with a cut-point at 547 days (six months into the follow-up period) were considered. The pre-start and pre-risk data points are useful for the purpose of programing at the analysis stage. For the Bayesian model, further data preparation involved putting the data into a format for use in WinBUGS. This was facilitated by the $d p u t$ command in $\mathrm{R}$.

\subsubsection{Analysis of Simulated Data Sets}

For both frequentist and Bayesian analysis, risk periods were defined as periods including 14 to 35 days after exposure to MMR vaccine, which reflects on the period it takes the virus to replicate $[4,5]$. Control periods were defined as all periods outside the risk period but within the follow-up period. Covariates included in the model were age adjusted at 6 months after the start of the observation period, which is at 1.5 years age of the child. The incidence rate ratio (IRR) for exposure to MMR and the respective 95\% Confidence (Credible) Intervals (CI) were obtained using the conditional Poisson regression model stratified by each case $[4,5]$. This is done by comparing incidents during the risk and control periods.
In the frequentist setting, in order to compare the results with those obtained by Whitaker, Paddy Farrington, Spiessens and Musonda [5], the analysis took up the same approach as [5]. The approach can also be found on their website at Open University [4].

For the Bayesian models fitted, samples of 15000 values were achieved after a burn-in of 1000 iterations from three chains. The chains were all started from dispersed initial points. Convergence of the MCMC to the target distribution was monitored via the density, trace, time series, autocorrelation function ( $a c f)$ plots and Gelman \& Rubin plots and statistic. Furthermore, the relationship between the MC error and the posterior standard deviation for the parameters of interest was also utilized.

\subsubsection{Prior Specification}

To incorporate the Bayesian approach, priors for the parameters in the model in equation (22) were specified. In the case of non-informative priors, a normal distribution with mean 0 and variance $1.0 \mathrm{E}-3(\operatorname{dnorm}(0,1.0 E-3))$ for the two parameters in the model was assumed. Similarly, a normal distribution was considered for the random effect $\zeta$, $\operatorname{dnorm}(0, t a u)$ with tau dgamma $(1,0.1)$. In the case of informative priors, the same distributions were assumed for the parameters but different mean and variances for the two parameters based on the information obtained from the literature $[5,31]$.

\subsection{Application to Oxford MMR Vaccine Data}

To illustrate the modelling approach discussed in sections 3 and 6 , the MMR data set previously described by $[5,31]$ on children who received the MMR vaccine at Oxford University hospital was used. A brief description of the data set is provided as follows.

The MMR data set consists of episodes of viral meningitis arising from children aged between 365 - 730 days over a calendar period of 1st October 1988 to 31st December 1991. Once identified, the cases were then linked to the vaccination records. This facilitated labelling of the child's follow-up time as exposed or not exposed to the vaccine. No information on the denominator was available as it was unclear what population was represented, since hospital catchment area where cases originated was not clearly defined. Cases were defined as children who had experienced at least one or more events of interest over the observation period. For the purpose of this analysis, each of the 10 cases identified had 
experienced only one episode of the outcome (viral meningitis). This study therefore considers a single event outcome as represented by Figure 1. Furthermore, Whitaker, Paddy Farrington, Spiessens and Musonda [5] sought to investigate if the Urabe strain (a particular type of live mumps vaccine) was associated with an increase in the risk of viral meningitis. The same is investigated in this study using the frequentist and Bayesian approach. For the analysis, data was initially managed and analysis carried out as described in section 6.1.3.

\section{Results and Discussion}

\subsection{Results}

In this section, the diagnostic plots (section 7.1.1) and the parameter estimates (section 7.1.2) are presented. Given the number of models fitted, Bayesian diagnostic results are presented for only two of the models fitted with informative priors. That is, the model for simulated data with population relative risk assumed to be 12.04 , and the model fitted on the Oxford data set. However, the diagnostic results for the other

\section{Dynamic trace}

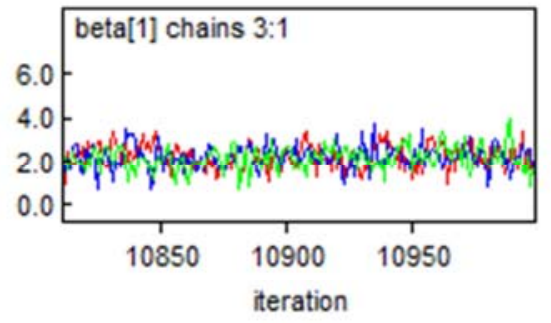

models (not presented) showed good convergence of the MCMC to the posterior distribution.

\subsubsection{MCMC Diagnostics}

The trace and time series plots in Figure 3 clearly show a good mix of the three chains considered. There is some very mild form of serial correlation between successive draws in the trace plots. Given the ACF plots in Figure 4, initially, autocorrelation is large at short lags but this quickly goes to zero. Brook-Gelman-Rubin statistic obtained was 1.05 which is very close to the recommended value of 1.1 . This confirms that convergence of the MCMC has been attained, and that a large portion of the samples being drawn are from distributions that are similar to the target distribution. Moreover, the effective sample size seem to be appropriate. Also, the ratio of the MC error to the posterior standard deviation for the parameters presented was less than the recommended cut off of $5 \%$. Similar plots were also witnessed with the other simulated data sets. The diagnostic plots and results for the MMR data set in Figure 5 and 6 also indicates a good mix of the three chains, and minimal serial correlation, hence suggesting good convergence.

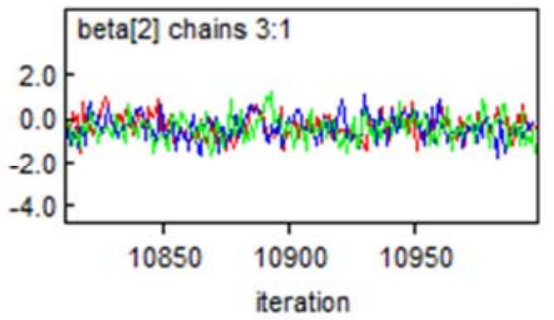

\section{Time series}
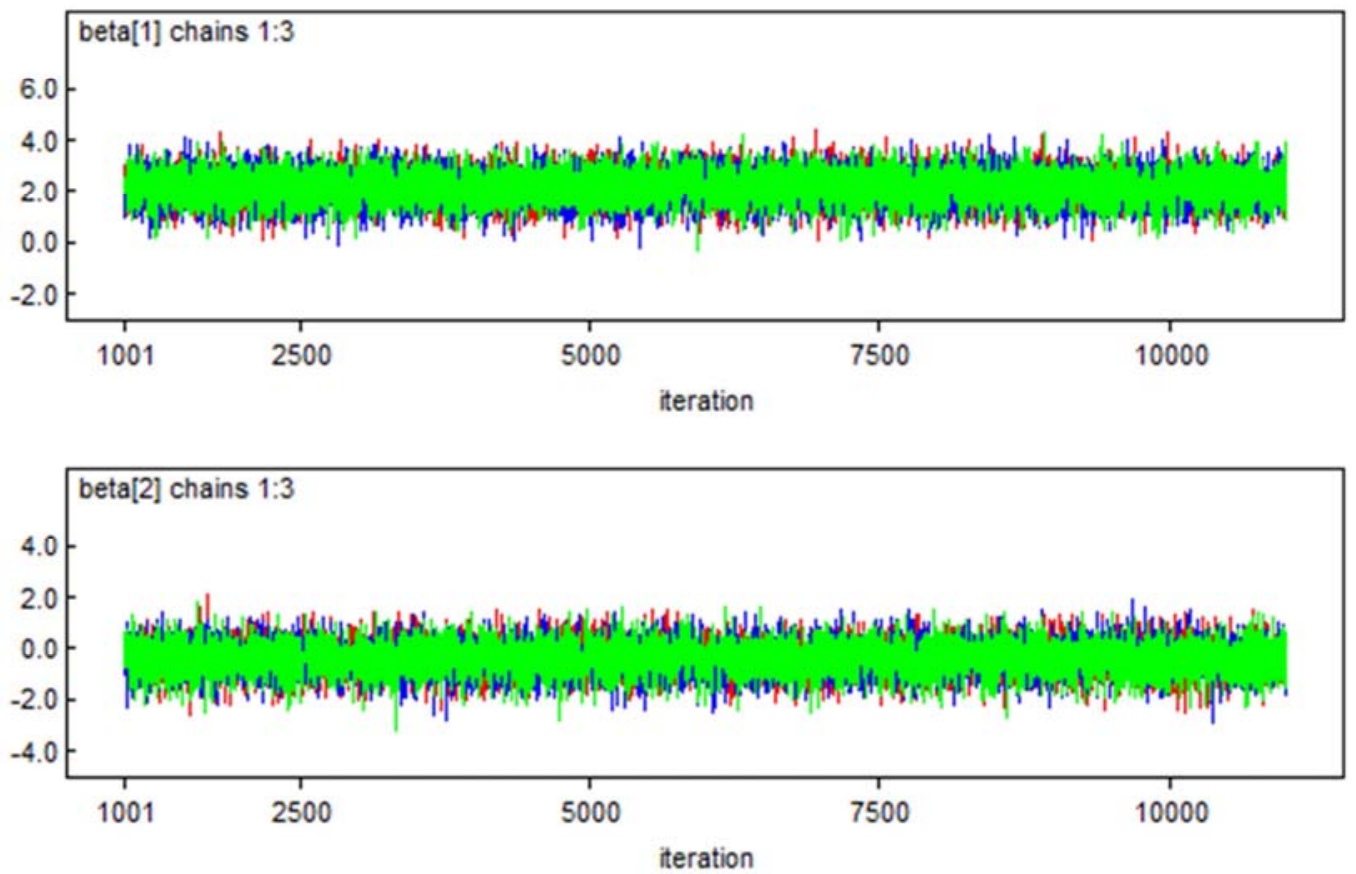

Figure 3. Trace and Time series plots of the parameters based on three chains for the simulated data of 10 children (IRR=12.04). 
Kernel density
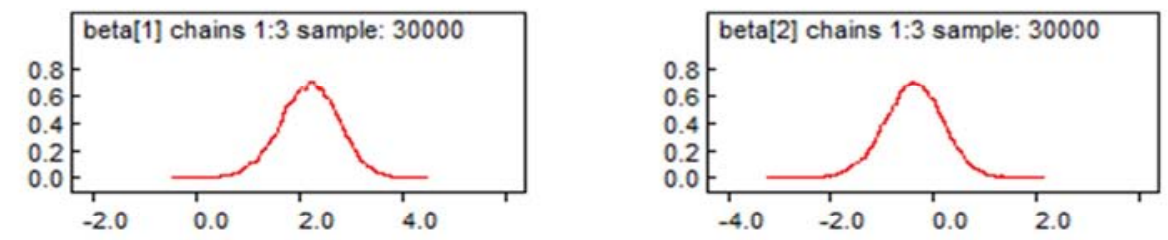

Gelman Rubin statistic
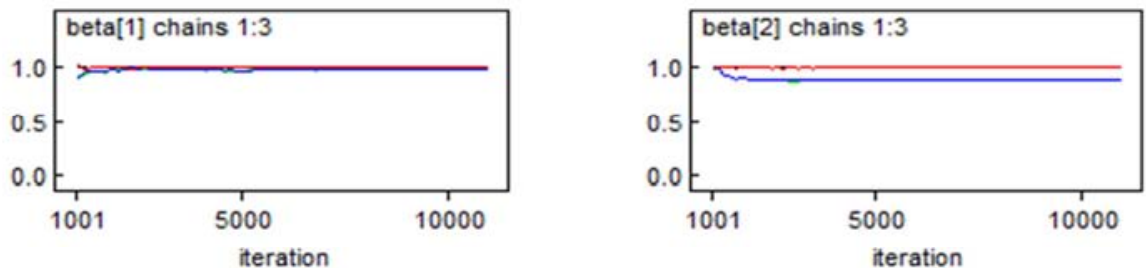

Autocorrelation function
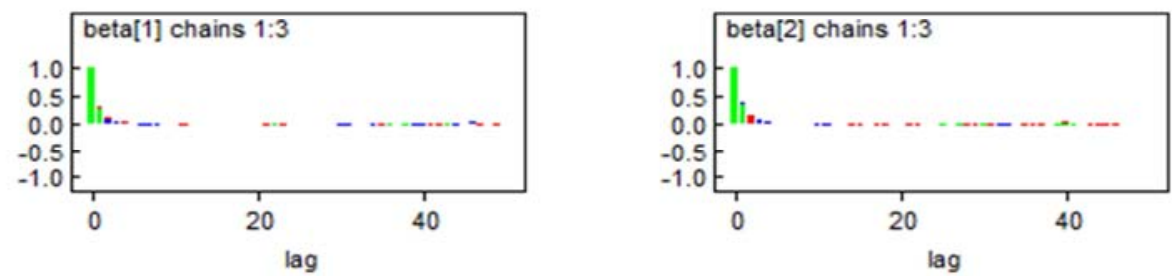

Figure 4. Density, Gelman \& Rubin, ACF plots for the simulated data set of 10 children (IRR=12.04).

Dynamic trace
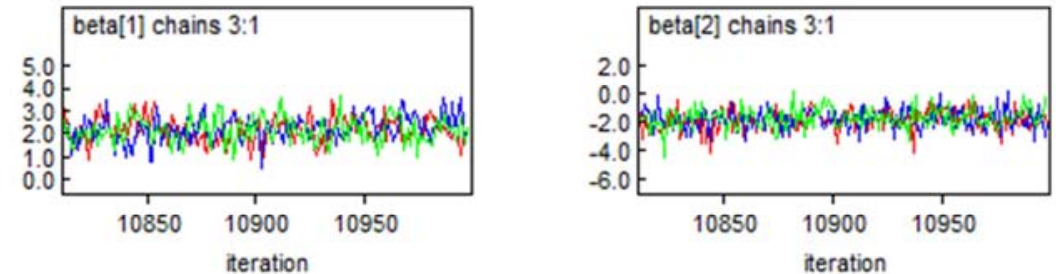

Time series
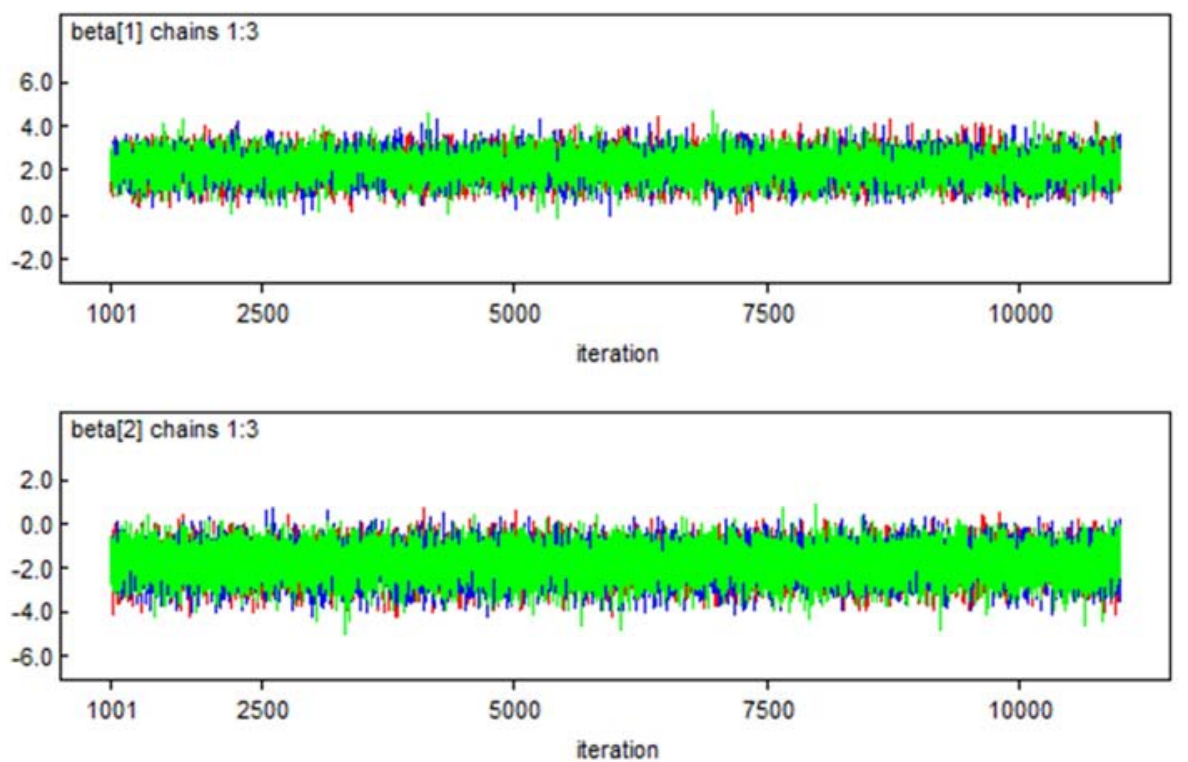

Figure 5. Trace and Time series plots of the parameters based on three chains for the Oxford MMR data. 
Kernel density
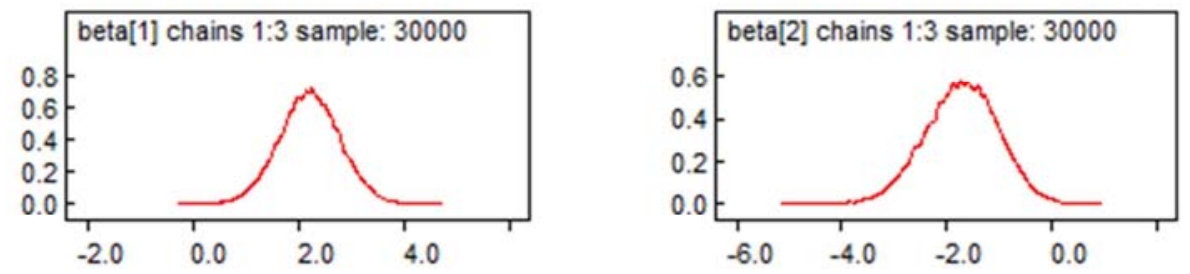

Gelman Rubin statistic
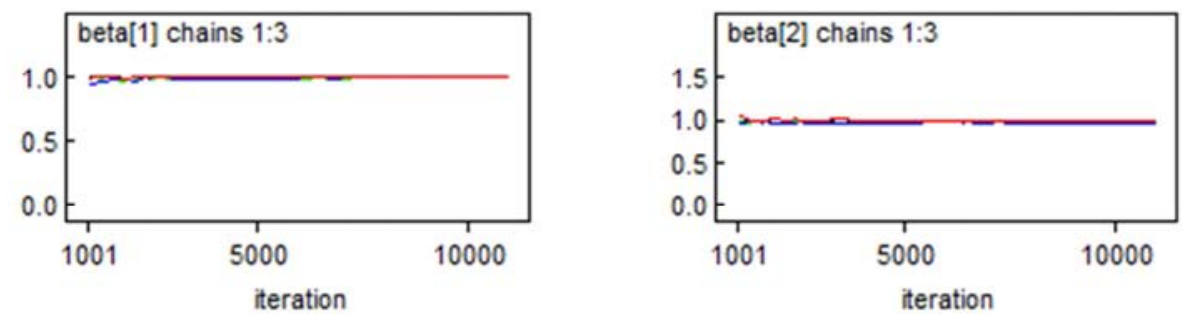

Astocorrelation function
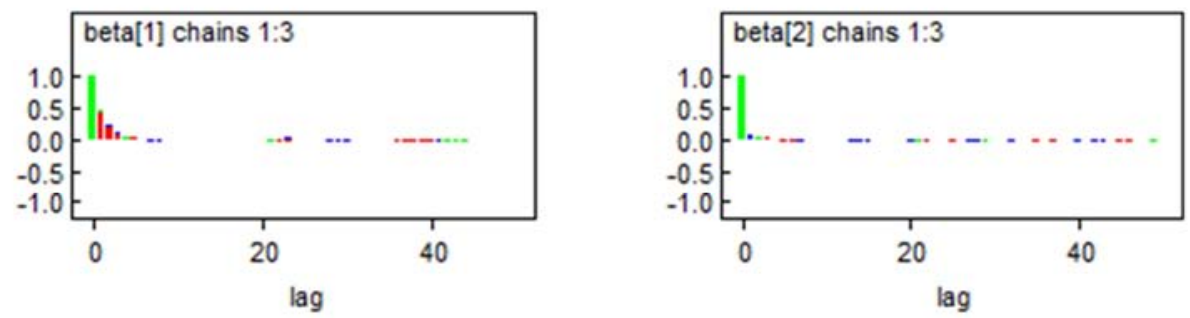

Figure 6. Density, Gelman \& Rubin, ACF plots based on three chains for the Oxford MMR data.

\subsubsection{Parameter Estimates}

\section{Simulated Data}

Table 2 presents the posterior estimates of the exposure (MMR vaccination $\left.-\beta_{1}\right)$ and age $\left(\beta_{2}\right)$ parameters of the Bayesian case-series model, and the classical model. The models were fitted on the data simulated assuming a population relative risk of 2.5 . For the three sample sizes used $(10,20,50)$, using a non-informative prior for Bayesian model, exposure to MMR vaccine was only associated with an increased risk of viral meningitis between 14-35 days post-vaccination only for the sample of 50 children. These results were true for both Bayesian IRR 95\% CI 2.878 (1.261 - 5.995) and frequentist 3.74 (1.73 8.05). However, the Bayesian model had a smaller interval. Similar pattern of results are also seen when an informative prior was used for the Bayesian model. Age seem to be giving similar and consistent results across all the models presented in Table 2 . In Table 3, the population relative risk assumed for simulating the data was 5.0. Significant associations are observed for samples of 10 and 50 but not for 20 with an uninformative prior. The same trend of results is also seen when informative priors are used. Compared to the frequentist model, similar pattern of results are witnessed. However, the frequentist estimates are far much higher than the Bayesian estimates in absolute terms. This shows a much higher association of viral meningitis with exposure to MMR vaccination in the 14-35 days post vaccination. Moreover, the Bayesian intervals are again seen to be smaller. For the final three simulated data sets of size 10, 20 and 50, the population relative risk was assumed to be 12.04 and the results of the fitted models are presented in Table 4. Results in this table show that the frequentist method seem to give lower values in absolute terms as compared to the Bayesian models both with informative and non-informative prior. However, the results are quite similar and the Bayesian model seem to give again smaller intervals. In all the three models fitted for various sample sizes, exposure to MMR vaccine was associated with a higher risk of viral meningitis within 14-35 days post vaccination. Age again is seen not to be associated with the outcome event, although it appears that increase in age is associated with lower risk of viral meningitis.

Application to the Oxford MMR data

Finally, Table 5 presents the result of the analysis carried out with the Oxford MMR vaccine data set. The table displays results of the proposed Bayesian model and the frequentist model as previously fitted by [5]. From the model diagnostic plots (Figures 5 and 6), the plots shows convergence of the underlying MCMC to stationarity. Results obtained from the frequentist model shows an increased risk of the outcome viral meningitis within between 14-35 days post vaccination with IRR 95\% CI 12.037 (3.002 - 48.259). For the Bayesian model, similar results were observed for the outcome viral meningitis given exposure to MMR vaccine, but the magnitude to the risk is reduced and also with a smaller credible interval, IRR 95\% 7.941 (2.104 - 30.908). The estimates were improved further when a more informative 
prior was used with a further shorter credible band of IRR 95\% CI 8.971 (2.869 - 27.994) being reported. Increase in age is significantly associated with reduced risk of meningitis in the BSCCS model IRR 0.177 (0.044-0.660).

Table 2. Posterior estimates of exposure and age for the age-adjusted Bayesian case-series model: Assumed IRR=2.5.

\begin{tabular}{|c|c|c|c|c|c|c|c|c|}
\hline Parameter & size & mean & sd & $2.5 \%$ & $97.5 \%$ & MC Error & IRR (95\% CI) & Freq. IRR (95\% CI) \\
\hline \multicolumn{9}{|l|}{ Non-Inf. prior } \\
\hline$\beta_{1} \dagger$ & 20 & 0.193 & 0.841 & -1.715 & 1.590 & 0.006 & $1.212(0.180-4.904)$ & $1.96(0.45-8.57)$ \\
\hline \multirow[t]{3}{*}{$\beta_{2} \dagger \dagger$} & 10 & -0.067 & 0.628 & -1.298 & 1.172 & 0.007 & $0.935(0.273-3.228)$ & $1.36(0.38-4.93)$ \\
\hline & 20 & -0.296 & 0.431 & -1.154 & 0.540 & 0.004 & $0.744(0.315-1.716)$ & $1.03(0.43-2.50)$ \\
\hline & 50 & 0.097 & 0.272 & -0.431 & 0.641 & 0.003 & $1.102(0.650-1.898)$ & $1.56(0.88-2.75)$ \\
\hline \multicolumn{9}{|l|}{ Inf. prior } \\
\hline \multirow[t]{3}{*}{$\beta_{1} \dagger$} & 10 & 1.310 & 0.654 & -0.044 & 2.536 & 0.005 & $3.706(0.957-12.629)$ & \\
\hline & 20 & 0.407 & 0.636 & -0.939 & 1.544 & 0.004 & $1.502(0.391-4.683)$ & \\
\hline & 50 & 1.077 & 0.381 & 0.292 & 1.785 & 0.003 & $2.936(1.339-5.960)$ & \\
\hline \multirow[t]{3}{*}{$\beta_{2} \dagger \dagger$} & 10 & -0.001 & 0.562 & -1.086 & 1.116 & 0.006 & $0.999(0.338-3.053)$ & \\
\hline & 20 & -0.260 & 0.418 & -1.094 & 0.558 & 0.004 & $0.771(0.335-1.747)$ & \\
\hline & 50 & 0.104 & 0.269 & -0.423 & 0.640 & 0.003 & $1.109(0.655-1.896)$ & \\
\hline$†$ MMR vaccin & $a+\dagger$ & & & & & & & \\
\hline
\end{tabular}

Table 3. Posterior estimates of exposure and age for the age-adjusted Bayesian case-series model: Assumed IRR=5.0.

\begin{tabular}{|c|c|c|c|c|c|c|c|c|}
\hline Parameter & size & mean & sd & $2.5 \%$ & $97.5 \%$ & MC Error & IRR $(95 \%$ CI) & Freq. IRR (95\% CI) \\
\hline \multicolumn{9}{|l|}{ Non-Inf. prior } \\
\hline \multirow[t]{3}{*}{$\beta_{1} \dagger$} & 10 & 2.198 & 0.825 & 0.513 & 3.799 & 0.007 & $9.007(1.670-44.657)$ & $11.34(2.41-53.18)$ \\
\hline & 20 & -0.988 & 1.309 & -4.120 & 0.988 & 0.008 & $0.372(0.016-2.685)$ & $0.79(0.11-5.97)$ \\
\hline & 50 & 1.317 & 0.360 & 0.577 & 1.990 & 0.003 & $3.732(1.781-7.316)$ & $4.76(2.36-9.61)$ \\
\hline \multirow[t]{3}{*}{$\beta_{2} \dagger \dagger$} & 10 & -1.571 & 0.790 & -3.274 & -0.161 & 0.007 & $0.208(0.038-0.851)$ & $0.30(0.07-1.36)$ \\
\hline & 20 & -1.162 & 0.494 & -2.192 & -0.240 & 0.004 & $0.313(0.112-0.786)$ & $0.42(0.16-1.10)$ \\
\hline & 50 & -0.257 & 0.275 & -0.800 & 0.288 & 0.003 & $0.773(0.449-1.334)$ & $1.04(0.59-1.83)$ \\
\hline \multirow[t]{3}{*}{$\beta_{1} \dagger$} & 10 & 2.243 & 0.638 & 0.963 & 3.481 & 0.005 & $9.422(2.620-32.492)$ & \\
\hline & 20 & -0.990 & 1.352 & -4.129 & 0.987 & 0.008 & $0.371(0.016-2.682)$ & \\
\hline & 50 & 1.328 & 0.350 & 0.610 & 1.985 & 0.002 & $3.773(1.840-7.279)$ & \\
\hline \multirow[t]{3}{*}{$\beta_{2} \dagger \dagger$} & 10 & -1.423 & 0.624 & -2.701 & -0.250 & 0.005 & $0.241(0.067-0.779)$ & \\
\hline & 20 & -1.163 & 0.496 & -2.192 & -0.240 & 0.004 & $0.313(0.112-0.787)$ & \\
\hline & 50 & -0.251 & 0.271 & -0.784 & 0.286 & 0.003 & $0.778(0.457-1.331)$ & \\
\hline$\dagger$ MMR vaccin & $n \dagger \dagger A$ & & & & & & & \\
\hline
\end{tabular}

Table 4. Posterior estimates of exposure and age for the age-adjusted Bayesian case-series model: Assumed IRR=12.04.

\begin{tabular}{|c|c|c|c|c|c|c|c|c|}
\hline Parameter & size & mean & sd & $2.5 \%$ & $97.5 \%$ & MC Error & IRR (95\% CI) & Freq. IRR (95\% CI) \\
\hline \multicolumn{9}{|c|}{ Non-Inf. prior } \\
\hline \multirow[t]{3}{*}{$\beta_{1} \dagger$} & 10 & 2.191 & 0.679 & 0.799 & 3.467 & 0.008 & $8.944(2.223-32.040)$ & $7.88(1.94-31.95)$ \\
\hline & 20 & 1.709 & 0.510 & 0.656 & 2.653 & 0.004 & $5.523(1.926-14.197)$ & $7.56(2.85-20.06)$ \\
\hline & 50 & 1.764 & 0.319 & 1.117 & 2.371 & 0.002 & $5.836(3.056-10.708)$ & $7.40(3.94-13.90)$ \\
\hline \multirow[t]{3}{*}{$\beta_{2} \dagger \dagger$} & 10 & -0.604 & 0.697 & -2.036 & 0.722 & 0.009 & $0.547(0.131-2.059)$ & $1.28(0.34-4.79)$ \\
\hline & 20 & -0.347 & 0.454 & -1.261 & 0.541 & 0.004 & $0.707(0.283-1.718)$ & $0.99(0.39-2.49)$ \\
\hline & 50 & -0.469 & 0.283 & -1.032 & 0.081 & 0.003 & $0.626(0.356-1.084)$ & $0.83(0.46-1.47)$ \\
\hline \multicolumn{9}{|l|}{ Inf. prior } \\
\hline \multirow[t]{3}{*}{$\beta_{1} \dagger$} & 10 & 2.177 & 0.578 & 1.011 & 3.279 & 0.005 & $8.820(2.748-26.549)$ & \\
\hline & 20 & 1.747 & 0.470 & 0.779 & 2.624 & 0.003 & $5.737(2.180-13.791)$ & \\
\hline & 50 & 1.771 & 0.314 & 1.127 & 2.368 & 0.002 & $5.877(3.086-10.676)$ & \\
\hline \multirow[t]{3}{*}{$\beta_{2} \dagger \dagger$} & 10 & -0.390 & 0.581 & -1.559 & 0.726 & 0.005 & $0.677(0.210-2.067)$ & \\
\hline & 20 & -0.314 & 0.435 & -1.184 & 0.535 & 0.004 & $0.730(0.306-1.708)$ & \\
\hline & 50 & -0.462 & 0.280 & -1.018 & 0.081 & 0.003 & $0.630(0.361-1.084)$ & \\
\hline \multicolumn{9}{|c|}{$\dagger$ MMR vaccination $\uparrow \dagger$ Age group } \\
\hline
\end{tabular}

Table 5. Bayesian Posterior estimates of the risk of hospital admission for aseptic meningitis from MMR vaccine using the Age-adjusted Bayesian case-series model - (30000 Simulations after 1000 burn-ins with three chains).

\begin{tabular}{lclllllll}
\hline Parameter & size & mean & sd & $\mathbf{2 . 5 \%}$ & $\mathbf{9 7 . 5 \%}$ & MC Error & IRR (95\% CI) & Freq. IRR (95\% CI) \\
\hline $\begin{array}{l}\text { Non-Inf. prior } \\
\beta_{1} \dagger\end{array}$ & 10 & 2.072 & 0.684 & 0.7436 & 3.431 & 0.0098 & $7.941(2.104-30.908)$ \\
$\beta_{2} \dagger \dagger$ & 10 & -2.365 & 1.352 & 0.0126 & -5.477 & -0.2057 & $0.094(0.004-0.814)$ & $0.225(0.251-2.016)$ \\
Inf. prior & & & & & & \\
$\beta_{1} \dagger$ & 10 & 2.194 & 0.577 & 1.054 & 3.332 & 0.0060 & $8.971(2.869-27.994)$ \\
\hline
\end{tabular}




\begin{tabular}{lllllllll}
\hline Parameter & size & mean & sd & $\mathbf{2 . 5 \%}$ & $\mathbf{9 7 . 5 \%}$ & MC Error & IRR (95\% CI) & Freq. IRR (95\% CI) \\
\hline $\begin{array}{l}\beta_{2} \dagger \dagger \\
\dagger \text { MMR vaccination } \dagger \dagger \text { Age group }\end{array}$ & -1.723 & 0.698 & -3.134 & -0.4157 & 0.0049 & $0.177(0.044-0.660)$ & \\
\hline
\end{tabular}

\subsection{Discussion}

In this study, the BSCCS model has been used to obtain posterior estimates of the model parameters under different conditions for viral meningitis as the outcome of interest. The risk of viral meningitis with exposure to MMR vaccine has been well defined in previous research $[26,32,33]$. The posterior estimates of the parameters obtained in this study demonstrates similar trend in risk, with exposure to MMR vaccine. These results indicate that the risk of viral meningitis increased up to nine-fold between 14 to 35 days after receiving the MMR vaccine. This study thus supports the results that have been previously presented by studies that have looked at the risk of meningitis in the second year of life, using the same data set or other data sets with different approaches [5, 31, 34, 35]. Similar results have also been obtained using the cross-over design, especially the Urabe strain, with risk being elevated from three to six weeks after vaccination [36]. However, this study further presents an improvement in terms of the point and the interval estimates. This is an addition to the methods available for use in vaccine safety surveillance. For simplicity, this study has only considered one exposure in the entire follow-up period. Further research is looking at using the model when an individual has more than one exposure period in the entire follow-up. Also, the choice of risk period has been guided by previous studies in order to enable comparison with results from previous studies [4, 5, 31]. Since the exact risk period is unknown, using more than one risk period, including time before exposure, would enable one to deal further with some of the very stringent assumptions of the SCCS model [5]. One of the advantages of the BSCCS is that even with small sample sizes, the SCCS method has been proved to be sufficient [37]. This is enhanced by the fact that Bayesian methods work well even with small sample sizes. Missing data can easily be handled in the analysis making the method to be more robust in obtaining the risk.

Despite these strengths of the method proposed, there was one case where results of the Bayesian model were higher than the results obtained by the frequentist approach. One of the possibilities is the presence of residual confounding. Age is adjusted for at the point of analysis but not incorporated at the point of data simulation. For actual data, this may not be an issue. Thus, the approach and results obtained in this study is currently being extended to consider other possible combinations in the follow-up period or pre-exposure periods. This will help in determining the possible bias due to incorrect specification of the risk periods and adjustment of the covariates under the BSCCS approach [38].

\section{Conclusion}

A Bayesian estimation approach for evaluating the risk of adverse events given transient exposure when the outcome event is left-truncated, has been proposed and evaluated. Simulation studies were used to illustrate the method, and an application to the real data has been demonstrated with results compared to those previously obtained using the frequentist approach. The method demonstrated a good performance compared to the frequentist approach that was previously used for the MMR data set. The model is applicable to a wide variety of cases where an exposure and outcome event occurs within an individual. Further work that is ongoing in this area involves modelling the risk of an outcome given multiple exposures and outcomes within an individual over the observation period, with different observation periods. One example is when an individual is on medication of more than one drug concomitantly, or a combination of drugs. Moreover, the ongoing work is also considering sensitivity analysis on the stringent assumptions of the case series model. These results, extend the advantage offered by the case-series method over other methods of analysis.

\section{Contributions}

HA, conceived and designed this study, conducted simulation analyses, interpreted the results, and drafted the manuscript. HA, AW, GO and SM were involved in discussing the design and analysis; critically revised the manuscript for its content and approved its final version.

\section{Ethical Approval}

This was a simulation study based on simulated patient records that used no human subjects. We determined that the study therefore did not require ethics committee approval. Our case study used data that was publicly available and has been approved for use in several studies.

\section{Acknowledgements}

Funding for the project was provided by Jomo Kenyatta University of Agriculture and Technology as part of postgraduate research.

\section{References}

[1] Barry, S. C. and T. J. O'Neill, The Bayesian Analysis of Truncated Regression Models. 1994.

[2] Johnson, N. L., A. W. Kemp, and S. Kotz, Univariate discrete distributions. Vol. 444. 2005: John Wiley \& Sons.

[3] Hardin, J. W. and J. M. Hilbe, Regression models for count data from truncated distributions. The Stata Journal, 2015. 15 (1): p. 226-246.

[4] Whitaker, H. J., The self-controlled case series method. 2005.

[5] Whitaker, H. J., C. Paddy Farrington, B. Spiessens, and P. Musonda, Tutorial in biostatistics: the self-controlled case series method. Statistics in medicine, 2006. 25 (10): p. 1768-1797. 
[6] David, F. N. and N. L. Johnson, The truncated poisson. Biometrics, 1952. 8 (4): p. 275-285.

[7] Tate, R. F. and R. L. Goen, Minimum variance unbiased estimation for the truncated Poisson distribution. The Annals of Mathematical Statistics, 1958: p. 755-765.

[8] Springael, L. and I. Van Nieuwenhuyse, on the sum of independent zero-truncated Poisson random variables. 2006.

[9] Shanker, R., F. Hagos, S. Sujatha, and Y. Abrehe, On zero-truncation of poisson and poisson-lindley distributions and their applications. Biometrics \& Biostatistics International Journal, 2015. 2 (6): p. 1-14.

[10] Yahaya, A., N. Dibal, A. Mobolaji, and G. Adegoke, Obtaining parameter estimate from the truncated Poisson probability distribution. 2016.

[11] Lee, C. H., Imprecise Prior for Imprecise Inference on Poisson Sampling Model. 2014.

[12] Gelman, A., J. B. Carlin, H. S. Stern, D. B. Dunson, A. Vehtari, and D. B. Rubin, Bayesian Data Analysis, Third Edition. 2013: Taylor \& Francis.

[13] Casella, G. and R. L. Berger, Statistical Inference. 2002: Duxbury Thomson Learning.

[14] Gamerman, D. and H. F. Lopes, Markov chain Monte Carlo: stochastic simulation for Bayesian inference. 2006: Chapman and Hall/CRC.

[15] Laksono, B. M., R. D. de Vries, S. McQuaid, W. P. Duprex, and R. L. de Swart, Measles Virus Host Invasion and Pathogenesis. Viruses, 2016. 8 (27483301): 210.

[16] Institute of Medicine, Adverse Effects of Vaccines: Evidence and Causality, ed. K. Stratton, et al. 2012: The National Academies Press.

[17] Stratton, K. R., C. J. Howe, and R. B. R. B. Johnston, Institute of Medicine; Measles and mumps vaccines, ed. K. R. Stratton, C. J. Howe, and R. B. R. B. Johnston. 1994: National Academy Press (US).

[18] World Health Organisation, Measles: Disease burden. 2020.

[19] Anderson, R. M., J. A. Crombie, and B. T. Grenfell, The epidemiology of mumps in the UK: a preliminary study of virus transmission, herd immunity and the potential impact of immunization. Epidemiology and infection, 1987. 99 (3609175): p. 65-84.

[20] Bennett, J. E., R. Dolin, and M. J. Blaser, Mandell, douglas, and bennett's principles and practice of infectious diseases: 2-volume set. Vol. 2. 2014: Elsevier Health Sciences.

[21] Mawson, A. R. and A. M. Croft, Rubella Virus Infection, the Congenital Rubella Syndrome, and the Link to Autism. International journal of environmental research and public health, 2019. 16 (31546693): 3543.

[22] Herrera, O. R., T. A. Thornton, R. A. Helms, and S. L. Foster, MMR Vaccine: When Is the Right Time for the Second Dose? The journal of pediatric pharmacology and therapeutics: JPPT: the official journal of PPAG, 2015. 20 (25964732): p. 144-148.

[23] Marlow, M. A., M. Marin, K. Moore, and M. Patel, CDC Guidance for Use of a Third Dose of MMR Vaccine During Mumps Outbreaks. Journal of Public Health Management and Practice, 2020. 26 (2).
[24] Su, S.-B., H.-L. Chang, Chen, and T. Kow, Current Status of Mumps Virus Infection: Epidemiology, Pathogenesis, and Vaccine. International journal of environmental research and public health, 2020. 17 (32150969): 1686.

[25] Furesz, J. and G. Contreras, Vaccine-related mumps meningitis-Canada. Canada diseases weekly report=Rapport hebdomadaire des maladies au Canada, 1990. 16: p. 253-4.

[26] Miller, E., M. Goldacre, S. Pugh, A. Colville, P. Farrington, A. Flower, J. Nash, L. MacFarlane, and R. Tettmar, Risk of aseptic meningitis after measles, mumps, and rubella vaccine in UK children. Lancet (London, England), 1993. 341: p. 979-82.

[27] Miller, E., P. Waight, C. P. Farrington, N. Andrews, J. Stowe, and B. Taylor, Idiopathic thrombocytopenic purpura and MMR vaccine. Archives of Disease in Childhood, 2001. 84 (3): p. 227-229.

[28] France, E. K., J. Glanz, S. Xu, S. Hambidge, K. Yamasaki, S. B. Black, M. Marcy, J. P. Mullooly, L. A. Jackson, J. Nordin, E. A. Belongia, K. Hohman, R. T. Chen, and R. Davis, Risk of Immune Thrombocytopenic Purpura After Measles-Mumps-Rubella Immunization in Children. Pediatrics, 2008. 121 (3): p. e687-e692.

[29] Center for Disease Control Prevention, Meningitis. 2020.

[30] Dubey, A. P. and S. Banerjee, Measles, mumps, rubella (MMR) vaccine. The Indian Journal of Pediatrics, 2003.70 (7): p. 579584.

[31] Weldeselassie, Y. G., H. Whitaker, and P. Farrington, SCCS: The Self-Controlled Case Series Method. 2019.

[32] Dourado, I., S. Cunha, M. d. G. Teixeira, C. P. Farrington, A. Melo, R. Lucena, and M. L. Barreto, Outbreak of Aseptic Meningitis associated with Mass Vaccination with a Urabe-containing Measles-Mumps-Rubella Vaccine: Implications for Immunization Programs. American Journal of Epidemiology, 2000. 151 (5): p. 524-530.

[33] Sugiura, A. and A. Yamada, Aseptic meningitis as a complication of mumps vaccination. Pediatr Infect Dis J, 1991. 10 (3): p. 209-13.

[34] Kamali Aghdam, M., M. Sadeghzadeh, S. Fakhimi, and K. Eftekhari, Evaluation of Aseptic Meningitis Following Measles-Mumps-Rubella Vaccine in Children Admitted due to Febrile Convulsion. International Journal of Pediatrics, 2018. 6 (8): p. 8147-8152.

[35] Park, T., M. Ki, and S. G. Yi, Statistical analysis of MMR vaccine adverse events on aseptic meningitis using the case cross-over design. Stat Med, 2004. 23 (12): p. 1871-83.

[36] Ki, M., T. Park, S. G. Yi, J. K. Oh, and B. Choi, Risk Analysis of Aseptic Meningitis after Measles-Mumps-Rubella Vaccination in Korean Children by Using a Case-Crossover Design. American Journal of Epidemiology, 2003. 157 (2): p. 158-165.

[37] Musonda, P., M. N. Hocine, H. J. Whitaker, and C. P. Farrington, Self-controlled case series analyses: Small-sample performance. Computational Statistics \& Data Analysis, 2008. 52 (4): p. 1942-1957.

[38] Campos, L. F., D. Şentürk, Y. Chen, and D. V. Nguyen, Bias and estimation under misspecification of the risk period in self-controlled case series studies. Stat (International Statistical Institute), 2017. 6 (1): p. 373-389. 